\title{
A New Approach to Fuzzy Multi-Criteria Acceptability Analysis
}

\author{
Boris Yatsalo \\ Information Systems Dept, National Research Nuclear University MEPHI (IATE NRNU MEPHI), \\ Studgorodok 1, Obninsk, Russian Federation, yatsalo@gmail.com
}

\begin{abstract}
The suggested approach, FMAA (Fuzzy Multicriteria Acceptability Analysis), assimilates uncertainties/ imprecisions of objective values and subjective judgments within the discrete Multi-Criteria Decision Analysis (MCDA). The presented algorithm for FMAA implementation utilizes fuzzy criterion values and fuzzy weight coefficients based on the use of fuzzy numbers, integral measure to assess the difference of fuzzy numbers, and determination of the fuzzy measure of "a Fuzzy Rank Statement", which is based on pairwise comparison of alternatives in an integrated scale. Implementation of FMAA based on one of the MCDA methods (MAVT) is discussed.
\end{abstract}

Keywords: Multi-Criteria Decision Analysis; Fuzzy numbers; Ranking fuzzy numbers; MAVT; DecernsMCDA.

\section{Introduction}

The practice of Multi-Criteria Decision Analysis (MCDA) faces with uncertainties/imprecisions of objective and subjective values of criteria and preference judgments. For analysis of various uncertainty types, including inaccuracy, randomness and imprecision, different multicriteria approaches and methods have been used [1-5].

Within this paper, the discrete multicriteria analysis, MADM (Multi-Attribute Decision Making) is considered in which the finite set of explicitly given alternatives is analyzed; MODM (Multi-Objective Decision Making), where large or infinite number of implicitly given alternatives is examined, is not discussed here.

So far, most classical MADM methods [1-5], including MAVT/MAUT ((Multi-Attribute Value/Utility Theory), AHP (Analytic Hierarchy Process), TOPSIS (Technique for Order Preference by Similarity to the Ideal Solution), ELECTRE (ELimination Et Choix Traduisant la REalité), and PROMETHEE (Preference Ranking Organization METHod for Enrichment Evaluations) have been extended with fuzzy modifications [4-15], including implementation of indicated methods with the use of novel fuzzy sets, such as intuitionistic, Pythagorean, and hesitant fuzzy sets [16-22] as well as methods with fuzzy linguistic information for decision analysis [23-27].
This paper introduces a new method for Fuzzy Multicriteria Acceptability Analysis, FMAA. Acceptability analysis in this case means that the choice of alternative(s) and their ranking is based on the measure to be accepted. FMAA assimilates both fuzzy criterion values and fuzzy weight coefficients. The suggested algorithms for FMAA is based on forming the Fuzzy Rank Statements (FRSs) and determination of Fuzzy Rank Acceptability Indices (FRAIs) on the basis of a pairwise comparison of alternatives using a novel integral/area measure.

Assessment of FRAIs is associated with comparison and subsequent ranking fuzzy numbers. Comparison of fuzzy numbers is one of the key problems of fuzzy sets [28]. There are more than 30 methods for comparing and ranking fuzzy numbers. Some of them, including surveys, classification of ranking procedures, using combined probability-based methods as well as implementation of fuzzy ranking for different topical problems, including decisionmaking, are discussed in [28-33].

Here further details about the propeties of ranking fuzzy numbers based on FRAIs conception are not presented (this is the subject for a sepaprate work). We consider FRAIs implementation within FMAA for solving multicriteria/MADM problems.

FMAA may be considered as a fuzzy modification of the ProMAA (Probabilistic MAA) method [34,35], developed as a probabilistic approach to implementation of MAA (Multi-Criteria Acceptability Analysis). ProMAA is close to the SMAA (Stochastic MAA) method [36,37] in the use of the acceptability principle. But, they differ conceptually in the probabilistic approach to interpretation of weights and criterion values in ProMAA with proximate numerical assessments of corresponding analytical expressions for RAIs (Rank Acceptability Indices), against the stochastic implementation in SMAA with the use of Monte Carlo simulations, where for each simulation of weight coefficients (with the sum equals 1) and criterion values, a given classical MADM model (e.g., MAVT, [36,37]) is implemented with treatment of ranks for alternatives for each Monte Carlo iteration and subsequent statistical assessments of the RAIs.

FMAA method is based on analytical approach to definition of FRSs and FRAIs, numerical estimations of integrals when comparing two fuzzy numbers, and implementation of transformation methods for assessing functions of fuzzy numbers. Basic expressions of FMAA, analytical definition of the 
difference of two fuzzy numbers, and analytical expressions for FRAIs, are well consistent with human intuition and have evident interpretation.

In section 2, we present the basic algorithm of FRAA (Fuzzy Rank Acceptability Analysis). Implementation of FRAA for fuzzy extension of MAVT is briefly considered in section 3 along with the example of using FMAA within a case study on multicriteria location problem.

\section{Algorithm of Fuzzy Rank Acceptability Analysis (FRAA)}

Fuzzy number $\mathrm{Z}$ [38] is considered here as a convex normal and restricted fuzzy set in $\mathbb{R}$ with a continuous membership function $\mu_{z}(x)$ :

$$
\begin{gathered}
Z=\left\{\left(x, \mu_{Z}(x)\right): \mu_{Z}(x)>0,\right. \\
c_{1}<x<c_{2}, \mu_{Z}\left(c_{i}\right)=0, i=1,2 ; \\
\left.\mu_{Z}(x)=0, x \notin\left[c_{1}, c_{2}\right]\right\}
\end{gathered}
$$

For singleton $z=c, \mu_{Z}(c)=1$.

For fuzzy number $\mathrm{Z}$, a measure of membership of $\mathrm{Z}$ to the set of nonnegative fuzzy numbers, $\mathbb{F}_{\mathbb{R} \geq 0}$, may be defined with the use of its membership function $\mu_{z}(x)$ :

$$
\mu_{\mathbb{F}_{\mathbb{R} \geq 0}}(Z)=\int_{x \geq 0} \mu_{z}(x) d x / \int_{c_{1}}^{c_{2}} \mu_{z}(x) d x ;
$$

we assume, $\mathrm{c}_{1}<\mathrm{c}_{2}$; for any singleton $\mathrm{z}$ the measure of membership to $\mathbb{F}_{\mathbb{R} \geq 0}$ equals 1 for $\mathrm{z} \geq 0$ and 0 for $\mathrm{z}<0$. (The defined in (1) "measure of positivity" for fuzzy number $Z$ can also be derived with the use of its $\alpha$ cuts.)

For fuzzy numbers $Z_{1}$ and $Z_{2}$, consider $Z_{12}=Z_{1}-Z_{2}$, $Z_{21}=Z_{2}-Z_{1}$. We define, $Z_{1}$ exceeds $Z_{2}$ with the measure $\mu_{12}$ :

$$
\mu_{12}=\mu_{\mathbb{F}_{0+}}\left(Z_{12}\right)=\mu\left(Z_{1}-Z_{2} \geq 0\right)=\mu\left(Z_{1} \geq Z_{2}\right)
$$

Let us consider a Fuzzy Rank Statement (FRS), $S_{i k}$, for a given set of fuzzy numbers $\boldsymbol{Z}=\left\{\eta_{i}, \mathrm{i}=1, \ldots, n\right\}$ :

$$
S_{i k}=\left\{\eta_{i} \text { has the rank } k\right\}, i, k=1, \ldots, n
$$

(i.e., $k-1$ fuzzy numbers from the set $\mathbf{Z}$ can be considered as exceeding $\eta_{i}$ in the sense of a chosen measure). FRS $S_{i k}$ can be preented as follows:

$$
\begin{aligned}
& S_{i 1}=\bigwedge_{j \neq i}^{n}\left(\eta_{i} \geq \eta_{j}\right) \\
& S_{i 2}=\stackrel{n}{\underset{l \neq i}{\vee}}\left(\left(\eta_{i} \leq \eta_{l}\right) \underset{j \neq i, j \neq l}{\stackrel{n}{\wedge}}\left(\eta_{i} \geq \eta_{j}\right)\right) \\
& S_{i k}=\underset{\substack{\left(l_{1}<l_{2}<\ldots<l_{k-1}\right) \\
l_{s} \neq i, s=1, . ., k-1}}{\vee}\left((\overbrace{s=1}^{k-1}\left(\eta_{i} \leq \eta_{l_{s}}\right) \overbrace{\substack{j \neq i, j \neq l_{s} \\
s=1, \ldots, k-1}}^{n}\left(\eta_{i} \geq \eta_{j}\right))\right. \\
& F_{i n}=\widehat{j \neq i}^{n}\left(\eta_{i} \leq \eta_{j}\right) .
\end{aligned}
$$

FRAIs are defined as $\mu(i, k)=\mu\left\{S_{i k}\right\}$, and may be interpreted as a degree of confidence that alternative $a_{i}$ has the rank $k$. FRAIs can be calculated as follows based on fuzzy logic:

$$
\begin{aligned}
& \mu(i, 1)=\mu\left(S_{i 1}\right)=\mu\left(\widehat{(}_{j \neq i}^{n}\left(\eta_{i j} \geq 0\right)\right)= \\
& =\min _{j \neq i}\left\{\mu\left(\eta_{i j} \geq 0\right)\right\}=\min _{j \neq i}\left\{\mu_{i j}\right\}
\end{aligned}
$$

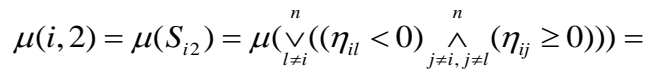

$$
\begin{aligned}
& =\max _{l \neq i}\left\{\min _{j \neq i, j \neq l}\left\{\mu_{l i}, \mu_{i j}\right\}\right\} \\
& \mu(i, k)=\mu\left(S_{i k}\right)=
\end{aligned}
$$

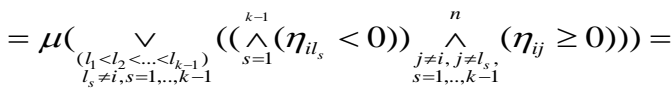

$$
\begin{aligned}
& =\max _{\substack{\left(l_{1}<l_{2}<\ldots<l_{k-1}\right) \\
l_{s} \neq i, s=1, \ldots, k-1}}\left\{\min \left\{\min _{s=1, . ., k-1} \mu_{l_{s} i}, \min _{\substack{j \neq i, j \neq l_{s} \\
s=1, \ldots, k-1}} \mu_{i j}\right\}\right\} \\
& \mu(i, n)=\mu\left(S_{i n}\right)=\min _{j \neq i}\left\{\left(1-\mu_{i j}\right)\right\}=\min _{j \neq i}\left\{\mu_{j i}\right\}
\end{aligned}
$$

Determined in (3) FRAIs can be used, e.g., for the choice of the biggest fuzzy number from the set of $n$ fuzzy numbers $\boldsymbol{Z}$, choosing number $i$ which maximizes $\mu(i, 1)$, etc. (details and properties of the introduced measure for comparison of fuzzy numbers and subsequent ranking are not considered in this paper and are a subject for a separate paper).

Thus, FRAA is based on implementation of FRAIs for selecting the "best" fuzzy number(s) and their ranking.

\section{Implementation of FMAA}

Fuzzy Multicriteria Acceptability Analysis, FMAA, is presented below (as an example of implementation) for a fuzzy extension of one of the classical MADM methods, MAVT, with subsequent implementation of FRAA.

The following additive MAVT model is considered:

$$
\begin{gathered}
V(\boldsymbol{a})=w_{1} V_{1}\left(a_{1}\right)+\ldots+w_{m} V_{m}\left(a_{m}\right), \\
w_{j}>0, \sum w_{j}=1,
\end{gathered}
$$

where $a_{j}$ is the criterion value for the alternative $\boldsymbol{a}$ for criterion $j, V_{j}(x)$ is a partial value function, $w_{j}$ is a criterion weight (reflecting a scaling factor), $j=1, \ldots, m$, $V(\boldsymbol{a})$ is interpreted as an integral/entire value of the alternative $\boldsymbol{a}[1-3]$

FMAVT (Fuzzy MAVT) is an extension of additive MAVT with a possibility to use fuzzy criterion values and fuzzy weights presented by fuzzy numbers.

Within FMAA, criterion values $a_{i j}=Z_{j}\left(\boldsymbol{a}_{i}\right)$ and weights $w_{j}$ are considered as fuzzy numbers, $i=1, \ldots, n$, $j=1, \ldots, m$, and overall (fuzzy) integrated value $V\left(\boldsymbol{a}_{i}\right)$ is determined according to (4) based on implementation of functions of fuzzy numbers [38] (setting fuzzy weights within FMAVT is considered below).

Let $\eta_{i}=V\left(\boldsymbol{a}_{i}\right)$ be the entire fuzzy value (4) of the alternative $\boldsymbol{a}_{i}$, and $\eta_{i j}=\eta_{i}-\eta_{j}, i, j=1, \ldots, n$. According to (3), the measure $\mu(i, k)=\mu\left\{S_{i k}\right\}$ of the FRS $S_{i k}=\{$ alternative $i$ has the $\operatorname{rank} k\}, i, k=1, \ldots, n$, is determined.

Using matrix $\mu(i, k)=\mu\left(S_{i k}\right), \quad i, k=1, \ldots n$, experts/ Decision-Makers can make a choice of the "most acceptable" alternative (which maximizes 
$\mu(i, 1))$, or ranking alternatives, if appropriate (maximizing $\mu(i, k)$ for the rank $k$ ).

The recommended approach for assigning fuzzy weights $w_{j}$ in FMAVT is a modification of the swing weighting process for MAVT [1-3] and can be briefly presented as follows:

Weight coefficient $w_{1}=1$ is assigned for the most highly weighted criterion (let us denote this criterion as $C_{1}$ ), taking into account evaluation of increase in overall value as a result of swing from worst to best for each criterion (taking into account the $\min c_{1, i}$ and $\max c_{2, i}$, see definition of fuzzy numbers in section 2 , for each criterion among all alternatives $i=1, \ldots, n)$;

- The variation interval for weight coefficient $w_{2}$, $\left[w_{2}^{\min }, w_{2}^{\max }\right], 0<w_{2}^{\min } \leq w_{2}^{\max } \leq 1$, is assigned for the second ranked criterion (we denote it as $C_{2}$ ) based on evaluation of a range for relative value of a swing from worst to best for this criterion in comparison with the corresponding value of swing for the most highly weighted criterion.

- Previous step is repeated for the third and subsequent criteria;

The membership function $\mu_{j}(x)$ for weight $w_{j}$, as for fuzzy number, is assigned by experts in the given interval $\left[w_{j}^{\min }, w_{j}^{\max }\right], j=2, \ldots, m$.

FMAA on the basis of FMAVT method (2)-(4), FMAVT-FMAA, was implemented in the Decision Support System (DSS) DecernsMCDA [39].

The version of FMAVT-FMAA, implemented in DecernsMCDA, is based on transformation method [40] (for assessing entire fuzzy value $V(\boldsymbol{a})$ in accordance with (4)) and numerical assessment of integrals (for estimating area measures in (1)). The current version of FMAVT-FMAA implemented in DecernsMCDA has the following features:

- User/experts can set for criterion values and weight coefficients the following types of fuzzy numbers:

- crisp,

- triangular,

- trapezoidal, and

- piecewise-linear;

- Different types of (monotonic) partial value functions may be used:

- linear,

- piecewise-linear, and

- non-linear/exponential.

- Implementation of value function sensitivity analysis (changing value function by mouse with effective graphical demonstration of changing the matrix of rank acceptability indices).

In addition, users/experts may analyze different scenarios for analysis of a given MCDA problem with the use of FMAVT-FMAA (i.e., different scenarios for weight coefficients and different partial value functions) and compare the outputs, if possible, with corresponding outputs for other MCDA methods implemented in DecernsMCDA (MAVT, TOPSIS, AHP, PROMETHEE, MAUT, ProMAA, FMAVT) [39], Fig.1.

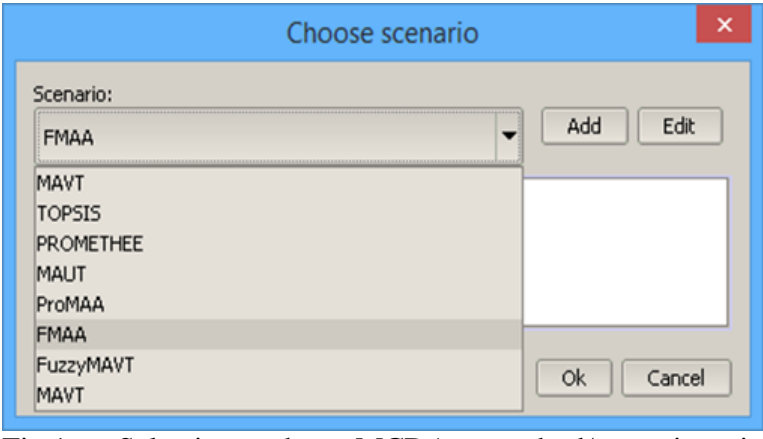

Fig.1: Selecting the MCDA method/scenario in DecernsMCDA.

Implementation of DecernsMCDA for risk management problems have been considered in $[41,42]$. To demonstrate the implementation of FMAVT-FMAA, we present here only some its outputs within a multicriteria housing development problem [41]; the use of different MCDA methods, including FMAVT-FMAA, for analysis of this problem with subsequent comparison of the output results, has been considered in details in [39].

Within this multicriteria problem, 5 criteria and 5 alternatives are analyzed on the step of the multicriteria decision process. For assessing criteria, GIS-functions from DecernsSDSS tools [39,41] were used (for 3 distance-based criteria) along with expert and economic evaluation (for 2 criteria) [41]. Eight MADM methods, incorporated in Decerns, including FMAA (FMAVT-FMAA), were used to select the "best" alternative.

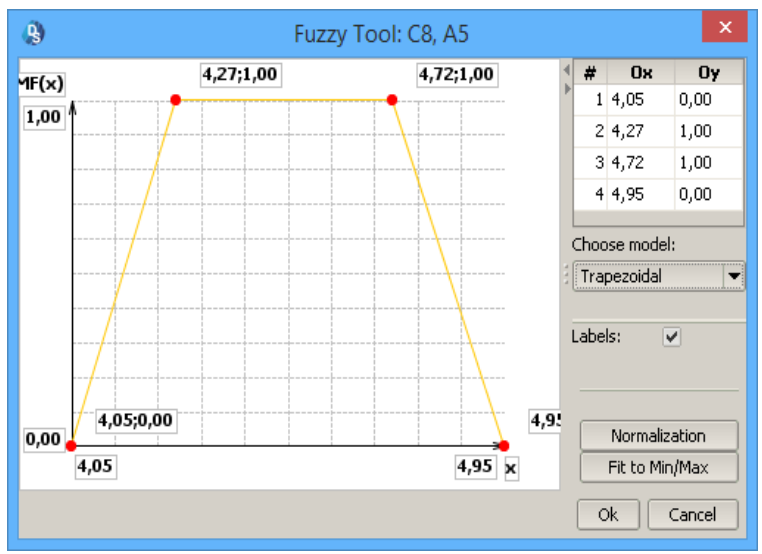

Fig.2: Setting fuzzy criterion value in DecernsMCDA

Fuzzy criterion values and weight coefficients (see Fig.2) as well as partial value functions were discussed with experts. The calculated FRAIs for this multicriteria problem are presented in Fig.3. The results of ranking alternatives according to different MCDA methods [39] are presented in Table 1. 


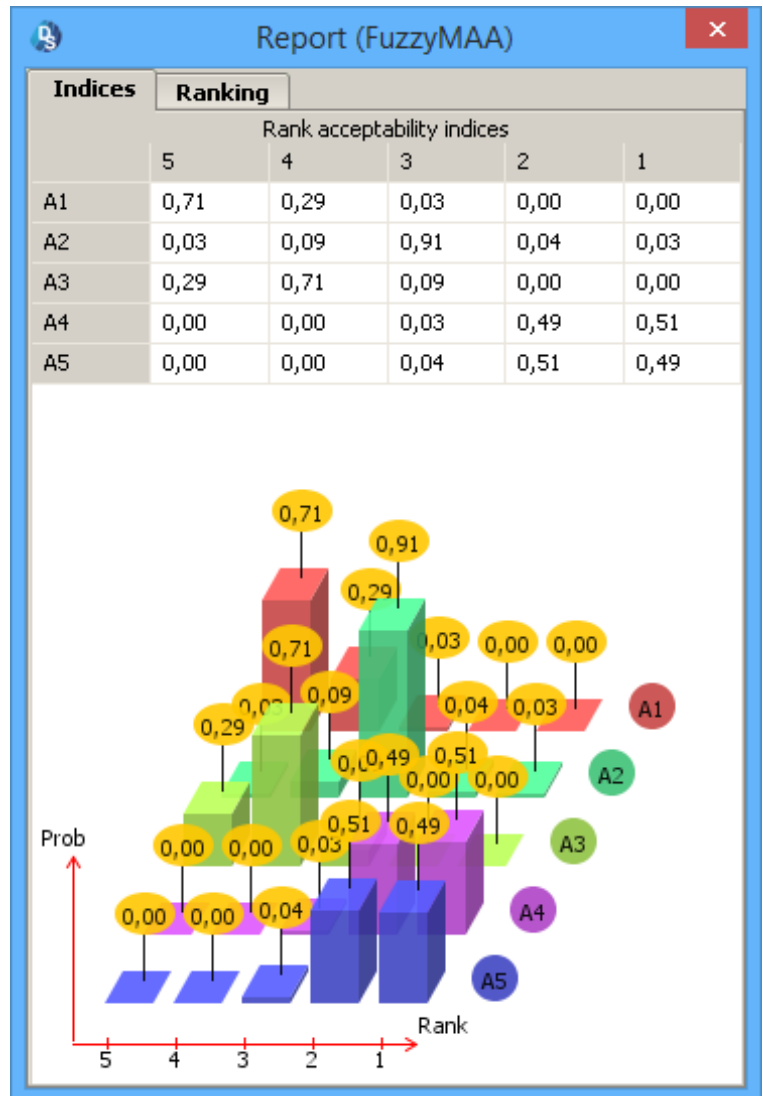

Fig.3: FRAIs for FMAVT-FMAA: output form in DecernsMCDA.

Table 1. Ranks of alternatives $A_{1}-A_{5}$ for different MCDA methods.

\begin{tabular}{|c|c|c|c|c|c|}
\hline $\begin{array}{c}\text { Method/ } \\
\text { Alternative }\end{array}$ & A1 & A2 & A3 & A4 & A5 \\
\hline MAVT & 5 & 3 & 4 & 2 & 1 \\
\hline TOPSIS & 5 & 3 & 4 & 1 & 2 \\
\hline $\begin{array}{c}\text { PROMETHEE } \\
\text {-I }\end{array}$ & 5 & 3 & 4 & $1-2$ & $1-2$ \\
\hline $\begin{array}{c}\text { PROMETHEE } \\
\text {-II }\end{array}$ & 5 & 3 & 4 & 2 & 1 \\
\hline MAUT & 5 & 3 & 4 & 2 & 1 \\
\hline ProMAA & 5 & 3 & 4 & 2 & 1 \\
\hline FMAA & 5 & 3 & 4 & 2 & 1 \\
\hline FMAVT & 5 & 3 & 4 & 2 & 1 \\
\hline
\end{tabular}

Even though, different MCDA methods may give different results in ranking alternative (e.g., see the results for MAVT and TOPSIS, Table 1), but for "relatively robust situation" corresponding results for choice and ranking multicriteria problems are identical or "relatively close", as we have in Table 1. FMAA-based ranking for this multicriteria problem is in agreement with the results for other MCDA methods. This (along with such analyses for other multicriteria problem) may serve as a confirmation of FMAA conception (implemented in FMAVT-FMAA method).

\section{Conclusions}

In this paper, an original and novel method, FMAA (Fuzzy Multicriteria Acceptability Analysis), is presented. FMAA is based on FRAIs (Fuzzy Rank Acceptability Indices) for assessment of the fuzzy measure of ranks for all the alternatives under consideration. Implementation of FMAA for a fuzzy extension of MAVT, FMAVT-FMAA, is described. This method has been implemented in the Decision Support System DecernsMCDA [39] and allows treating uncertainty of criterion values and weight coefficients based on the use of fuzzy numbers.

The method for assessing FRAIs based on FRSs (Fuzzy Rank Statements) (2), (3) seems, at first sight, as a complicated one. However, in fact, this method is well consistent with human intuition and has easy interpretation.

Realization of FMAVT-FMAA uses numerical methods for assessing area measures, transformation method for determining functions of fuzzy variables, and original algorithm for effective and fast calculation of FRAIs following fuzzy logic and based on binary coding.

The use of FMAVT-FMAA along with other methods, incorporated in DecernsMCDA, demonstrates adequacy of FMAA for analysis of wide range of multicriteria problems.

Implementation of FMAA conception based on some other MADM methods is also prospective as well as an adjustment of FMAA concept to other types of fuzzy sets.

\section{References}

[1] R.L. Keeney and H. Raiffa, Decision with Multiple Objectives (J. Wiley \& Sons, New York, 1976).

[2] D. von Winterfeldt and W. Edwards, Decision Analysis and Behavioral Research (Cambridge: Cambridge University Press, 1986).

[3] V. Belton and T. J. Stewart, Multiple Criteria Decision Analysis: An Integrated Approach (Kluwer Academic Publishers, 2002).

[4] J. Figueira, S. Greco and M. Ehrgott (eds), Multiple Criteria Decision Analysis: State of the Art Surveys (Springer, New York, 2005).

[5] A. Ishizaka and Ph. Nemery, Multi-criteria Decision Analysis: Methods and Software (WILEY, New York, 2013).

[6] O. Taylan, A.O. Bafail, R.M.S. Abdulaal and M.R. Kabli, Construction projects selection and risk assessment by fuzzy AHP and fuzzy TOPSIS methodologies, Applied Soft Computing, 17 (2014) 105-116.

[7] F.R. Lima Junior, L. Osiro and L.C.R. Carpinetti, A comparison between Fuzzy AHP and Fuzzy TOPSIS methods to supplier selection, Applied Soft Computing, 21 (2014) 194-209.

[8] T. Gürbüz, Y.E. Albayrak and E. Alaybeyoğlu, Criteria Weighting and 4P's Planning in Marketing Using a Fuzzy Metric Distance and AHP Hybrid 
Method, International Journal of Computational Intelligence Systems, 7(1) (2014) 94-104.

[9] H. Deng, C.H. Yeh and R. J. Willis, Intercompany comparison using modified TOPSIS with objective weights, Computers and Operations Research, 27(10) (2000) 963-973.

[10] C. Kahraman, N. Yasin, A. Sezi, Ç.M. Gülbay and S.A. Erdogan, Hierarchical fuzzy TOPSIS model for selection among logistics information technologies, Journal of Enterprise Information Management, 20(2) (2007) 143 - 168. DOI:10.1108/17410390710725742.

[11] Y.-H. Chen, T.-Ch. Wang and Ch.-Y. Wu, Strategic decisions using the fuzzy PROMETHEE for IS outsourcing, Expert Systems with Applications, 38(10) (2011) 13216-13222.

[12] J.R. Yu, W.Y. Shing, Fuzzy analytic hierarchy process and analytic network process: An integrated fuzzy logarithmic preference programming, Applied Soft Computing, 13 (2013) 1792-1799.

[13] C. Kahraman (Ed), Fuzzy Multi-Criteria Decision Making. Theory and Applications with Recent Developments (Springer, Series: Optimization and its Applications, 16, 2008).

[14] A. Jiménez, A. Mateos and P. Sabio, Dominance intensity measure within fuzzy weight oriented MAUT: An application, Omega, 41(2) (2013) 397-405.

[15] O.M. Ashour and G.E.O. Kremer, A simulation analysis of the impact of FAHP-MAUT triage algorithm on the Emergency Department performance measures, Expert Systems with Applications, 40(1) (2013) 177-187.

[16] M.C. Wu, T.Y. Chen, The ELECTRE multicriteria analysis approach based on Atanassov's intuitionistic fuzzy sets. Expert Systems with Applications, 2011. 38(10), 12318-12327.

[17] F. Shen, J. Xu, Z. Xu, An automatic ranking approach for multi-criteria group decision making under intuitionistic fuzzy environment. Fuzzy Optimization and Decision Making, 2015. DOI 10.1007/s10700-014-9201-5.

[18] X. Zhang, Z. Xu, Soft computing based on maximizing consensus and fuzzy TOPSIS approach to interval-valued intuitionistic fuzzy group decision making, Applied Soft Computing 26 (2015) 42-56.

[19] X. Zhang, Z. Xu, Extension of TOPSIS to Multiple Criteria Decision Making with Pythagorean Fuzzy Sets. International Journal Of Intelligent Systems, 29 (2014) 1061-1078.

[20] F. Herrera, L. Martınez, V. Torra and Z. Xu, Hesitant Fuzzy Sets: An Emerging Tool in Decision Making, International Journal of Intelligent Systems, 29 (2014) 493-494.

[21] N. Chen, Z. Xu, Hesitant fuzzy ELECTRE II approach: A new way to handle multi-criteria decision making problems. Information Sciences, 292 (2015) 175-197.
[22] S.C. Onar, B. Oztaysi and C. Kahraman, Strategic Decision Selection Using Hesitant fuzzy TOPSIS and Interval Type-2 Fuzzy AHP: A case study, International Journal of Computational Intelligence Systems, (Published online: $23 \mathrm{Sep}$ ) (2014).

[23] Ch.-T. Chen, P-F. Pai and W.-Zh. Hung, An Integrated Methodology using Linguistic PROMETHEE and Maximum Deviation Method for Third-party Logistics Supplier Selection, International Journal of Computational Intelligence Systems, 3(4) (2010) 438-451.

[24] Wu-E. Yanga, J.-Q. Wanga, X.-F. Wanga, An outranking method for multi-criteria decision making with duplex linguistic information, Fuzzy Sets and Systems, 198 (2012): 20-33.

[25] R.M. Rodriguez, L. Martınez, and F. Herrera, Hesitant Fuzzy Linguistic Term Sets for Decision Making, IEEE Transactions On Fuzzy Systems, 20(1) (2012) 109-119.

[26] B. Esteban et al., TPLUFIB-WEB: A fuzzy linguistic Web system to help in the treatment of low back pain problems, Knowl. Based Syst. (2014), http://dx.doi.org/10.1016/j.knosys.2014.03.004.

[27] F.J. Estrella, M. Espinilla, F. Herrera, L. Martínez, FLINTSTONES: A fuzzy linguistic decision tools enhancement suite based on the 2-tuple linguistic model and extensions, Information Sciences, 280 (2014) 152-170.

[28] B. Matarazzo, G. Munda, New approaches for the comparison of L-R fuzzy numbers: a theoretical and operational analysis, Fuzzy Sets Syst. 118 (2001) 407-418.

[29] C. Kahraman, A.C. Tolga, An Alternative Ranking Approach and Its Usage in Multi-Criteria Decision-Making, International Journal of Computational Intelligence Systems, 2(3) (2009) 219-235.

[30] S.H. Nasseri, F.Taleshian, Z. Alizadeh and J. Vahidi, Journal of Mathematics and Computer Science, 4(3) (2012) $283-294$.

[31] X. Wang, E.E. Kerre, Reasonable properties for the ordering of fuzzy quantities (I), Fuzzy Sets Syst., 118 (2001) 375-385.

[32] X. Wang, E.E. Kerre, Reasonable properties for the ordering of fuzzy quantities (II), Fuzzy Sets Syst., 118 (2001) 387-405.

[33] F. Zhang, J. Ignatius, Ch.P. Lim and Y. Zhao, A new method for ranking fuzzy numbers and its application to group decision making, Applied Mathematical Modelling, 38(4) (2014) 1563-1582.

[34] B. Yatsalo, S. Gritsyuk, A. Tkachuk, O. Mirzeabasov, Multicriteria Acceptability Analysis: ProMAA Approach, Proc. of the 25th Mini-EURO Conference "Uncertainty and Robustness in Planning and Decision Making, URPDM 2010". University of Coimbra, Portugal, 15-17 April, 2010. ISBN 978-989-95055-3-7. 
[35] B. Yatsalo, S. Gritsyuk, O. Mirzeabasov, M.Vasilevskaya, Uncertainty Treatment within Multicriteria Decision Analysis with the Use of Acceptability Concept, Upravlenie Bolshimi Sistemami, 32 (2011) 5-30 (in Russian).

[36] R. Lahdelma, J. Hokkanen, P. Salminen, SMAA - Stochastic Multiobjective Acceptability Analysis, European Journal of Operational Research 106 (1998) 137-143.

[37] T. Tervonen, J.R. Figueira, A Survey on Stochastic Multicriteria Acceptability Analysis Methods, Journal of Multi-Criteria Decision Analysis, 15 (2008) 1-14.

[38] K.H. Lee, First Course on Fuzzy Theory and Applications, (Springer-Verlag, BerlinHeidelberg, 2005).

[39] B. Yatsalo, V. Didenko, S. Gritsyuk, and T. Sullivan, Decerns: A Framework for MultiCriteria Decision Analysis, International Journal of Computational Intelligence Systems, 8(3) (2015) 467-489.

[40] M. Hanss, Applied Fuzzy Arithmetic (SpringerVerlag, Berlin-Heidelberg, 2005).

[41] B. Yatsalo, V. Didenko, A. Tkachuk, S. Gritsyuk, O.Mirzeabasov, A.Babutski, I.Pichugina, T.Sullivan and I.Linkov. MultiCriteria Spatial Decision Support System DECERNS: Application to Land Use Planning, International Journal of Information Systems and Social Change, 1(1) (2010) 11-30.

[42] B. Yatsalo, T. Sullivan, V.Didenko, S.Gritsyuk, O.Mirzeabasov, I.Pichugina and I.Linkov, Environmental risk management with the use of multi-criteria spatial decision support system DECERNS, International Journal of Risk Assessment and Management, 16(4) (2012) 175198. 